\title{
Chamber Hypertrophy or Enlargement ECG Assessment
}

National Cancer Institute

\section{Source}

National Cancer Institute. Chamber Hypertrophy or Enlargement ECG Assessment. NCI

Thesaurus. Code C111155.

An electrocardiographic assessment of chamber hypertrophy or enlargement. 